\title{
Neuromodulators Are Effective for Treatment of Spastic Chest Pain After Heller Myotomy for Achalasia
}

TO THE EDITOR: Chest pain (CP) affects $50-70 \%$ patients with achalasia and is often refractory to treatment. ${ }^{1}$ Previous studies have reported persistent and even worsening of $\mathrm{CP}$ in patients undergoing Heller myotomy (HM) and up to one-third of patients with $\mathrm{CP}$ after $\mathrm{HM}$ have $\mathrm{CP}^{2}$ Neuromodulators such as tricyclic antidepressants (TCAs) and selective serotonin reuptake inhibitors (SSRIs) have previously been demonstrated to treat noncardiac $\mathrm{CP}$ and esophageal hypersensitivity in subjects without achalasia. ${ }^{3}$ We hypothesized treatment with neuromodulators to be effective in treating $\mathrm{CP}$ in patients with achalasia after HM. After institutional review board approval, patients with achalasia who were prescribed neuromodulators (TCAs/SSRIs) for CP after HM were included retrospectively from 2016-2021. All patients had esophageal manometry following $\mathrm{HM}$ that demonstrated absent contractility. Five patients underwent pre-treatment timed barium esophagogram that did not reveal obstruction. Pain was assessed per Eckardt score ranging from $0-3$ where $0=$ no pain, $1=$ occasional pain, $2=$ daily pain, and $3=$ pain with each meal. ${ }^{4}$ Response to treatment at first follow up after starting neuromodulator was recorded using numeric modified Likert scale (symptom relief score [SRS]) from 1 to 5 , where $1=$ much worse, $2=$ slightly worse, $3=$ no change, $4=$ slightly better, and $5=$ much better.

Six patients (4 females) with a median age of 20.5 (range 1647) years at time of $\mathrm{HM}$ were included (Table). $\mathrm{CP}$ was reported at a median time interval of 10.5 months (range 1-41) after HM. SSRIs and TCAs were started in $2(33.3 \%)$ and 4 patients (66.7\%), respectively. All 6 patients (100.0\%) reported improvement in CP with a median SRS of 5 (much better) with 3 patients (50.0\%) having complete resolution after a median follow-up of 282 (range 103-
428) days. All included patients opted to continue treatment with neuromodulators and were doing well at the last follow up. Patient No. 2 (47-year-old female) reported dry mouth with amitriptyline and was switched to escitalopram with maintained response.

$\mathrm{CP}$ occurring after $\mathrm{HM}$ is a poorly understood phenomenon for which no standardized treatment exists currently. In this retrospective study, neuromodulators were found to be a safe and effective for treatment of $\mathrm{CP}$ persisting after $\mathrm{HM}$ in patients with achalasia. Complete resolution of pain was seen in $50.0 \%$ of patients. Our results suggest modulation of pain sensitivity can be a safe and effective option for treatment of $\mathrm{CP}$ after $\mathrm{HM}$.

The pathophysiology of $\mathrm{CP}$ after $\mathrm{HM}$ remains unknown with recent studies showing no relationship between age and occurrence of $\mathrm{CP}$ in achalasia patients. De-novo occurring $\mathrm{CP}$ is observed in $8.3 \%$ patients undergoing $\mathrm{HM}^{2}$ No manometric parameters have been reported to correlate with $\mathrm{CP}$ in achalasia arguing against a "mechanical" pathophysiology. All of the patients in our study had an "aperistaltic" esophagus effectively ruling out esophageal spasms as a cause of $\mathrm{CP}$. We postulated the origin of $\mathrm{CP}$ to be related to altered pain perception and esophageal hypersensitivity similar to functional $\mathrm{CP}^{6}$ Esophageal tissue injury from repetitive ongoing insult in achalasia patients may have led to peripheral and/or central sensitization leading to $\mathrm{CP}$. Similar mechanisms have been reported in functional $\mathrm{CP}$ where the hypersensitivity may persist event after removal of the inciting stimulus is removed. ${ }^{6}$ Neuromodulators including TCAs and SSRIs are first-line treatment for functional $\mathrm{CP}$ by modulating hypersensitivity. This prompted us to treat our achalasia patients with $\mathrm{CP}$ after HM. Potential other causes of $\mathrm{CP}$, including acid reflux and $\mathrm{CP}$ as a result of myotomy itself, should 
be considered before initiating treatment with neuromodulators. Future research evaluating neuromodulators in prospective trials coupled with testing for esophageal hypersensitivity are needed to better manage this perplexing condition.

\section{Manik Aggarwal, ${ }^{1}$ John McMichael, ${ }^{2}$ Sudish C Murthy, ${ }^{3}$ Siva Raja ${ }^{3}$ and Scott L Gabbard ${ }^{2 *}$}

${ }^{1}$ Department of Internal Medicine, ${ }^{2}$ Digestive Disease and Surgery Institute, Cleveland Clinic, OH, USA; and ${ }^{3}$ Department of Thoracic and Cardiovascular Surgery, Heart, Vascular and Thoracic Institute,

Cleveland Clinic, OH, USA

1. Sasaki A, Obuchi T, Nakajima J, Kimura Y, Koeda K, Wakabayashi G. Laparoscopic Heller myotomy with Dor fundoplication for achalasia: long-term outcomes and effect on chest pain. Dis Esophagus 2009;23:284-289.

2. Wuller C, Bessell JR, Watson DI. Chest pain before and after laparo-

3. Nguyen TMT, Eslick GD. Systematic review: the treatment of noncardiac chest pain with antidepressants. Aliment Pharmacol Ther 2012;35:493-500.

4. Vaezi MF, Pandolfino JE, Yadlapati RH, Greer KB, Kavitt RT. ACG clinical guidelines: ciagnosis and management of achalasia. Am J Gastroenterol 2020;115:1393-1411.

5. Sasaki A, Obuchi T, Nakajima J, Kimura Y, Koeda K, Wakabayashi lasia: long-term outcomes and effect on chest pain. Dis Esophagus 2009;23:284-289.

6. Aziz Q, Fass R, Gyawali CP, Miwa H, Pandolfino JE, Zerbib F. Esophageal disorders. Gastroenterology 2016;150:1368-1379.

7. Nguyen TMT, Eslick GD. Systematic review: the treatment of noncardiac chest pain with antidepressants. Aliment Pharmacol Ther 2012;35:493-500.

\section{Financial support: None.}

\section{Conflicts of interest: None.}

Author contributions: Conceptualization and methodology: Manik Aggarwal, Sudish C Murthy, Siva Raja, and Scott L Gabbard; data curation and formal analysis: Manik Aggarwal and John McMichael; interpretation of data: Manik Aggarwal, Sudish C Murthy, Siva Raja, and Scott L Gabbard; drafting of manuscript: Manik Aggarwal and Scott L Gabbard; and review, editing, and final approval: all authors. scopic cardiomyotomy for achalasia. ANZ J Surg 2011;81:590-594. G. Laparoscopic Heller myotomy with Dor fundoplication for acha-

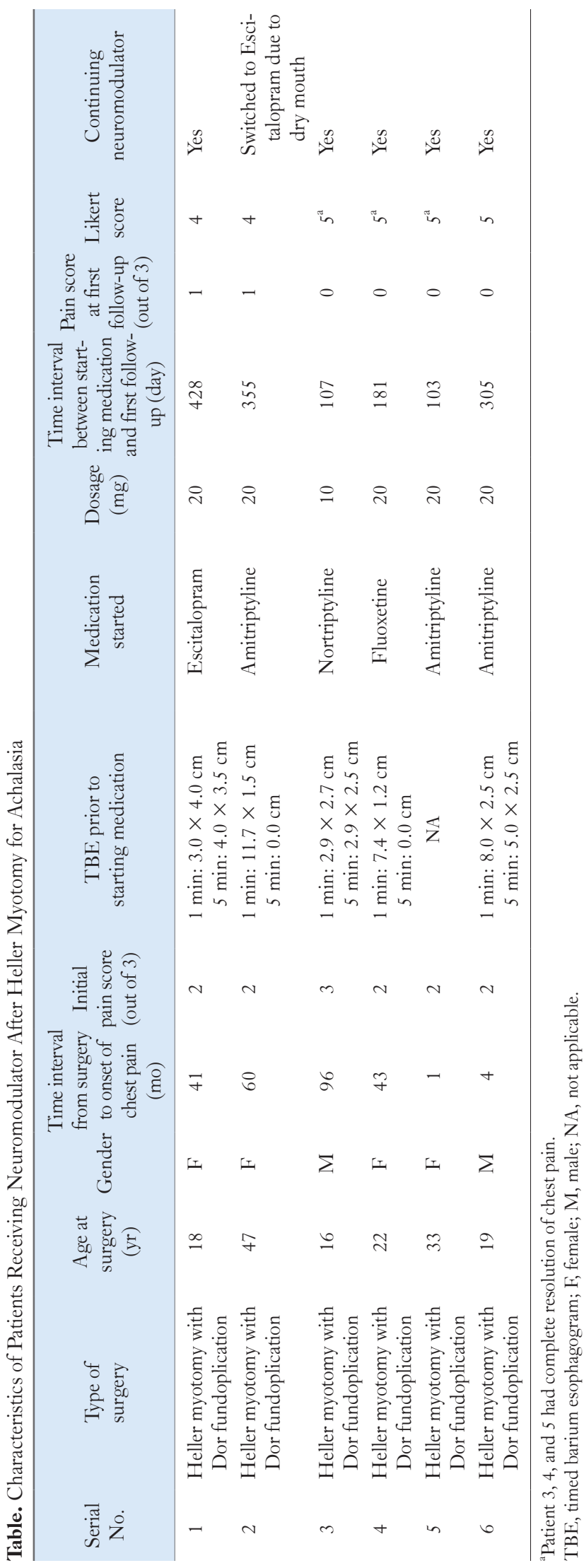

